This item was submitted to Loughborough's Research Repository by the author.

Items in Figshare are protected by copyright, with all rights reserved, unless otherwise indicated.

\title{
Accelerometer-measured daily steps and subjective cognitive ability in older adults: A two-year follow-up study
}

PLEASE CITE THE PUBLISHED VERSION

https://doi.org/10.1016/j.exger.2020.110874

\section{PUBLISHER}

Elsevier

\section{VERSION}

AM (Accepted Manuscript)

\section{PUBLISHER STATEMENT}

This paper was accepted for publication in the journal Experimental Gerontology and the definitive published version is available at https://doi.org/10.1016/j.exger.2020.110874.

\section{LICENCE}

CC BY-NC-ND 4.0

\section{REPOSITORY RECORD}

Chen, Shang-Ti, Clare Stevinson, Tian Tian, Li-Jung Chen, and Po-Wen Ku. 2020. "Accelerometer-measured Daily Steps and Subjective Cognitive Ability in Older Adults: A Two-year Follow-up Study”. Loughborough University. 
1 Title: Accelerometer-Measured Daily Steps and Subjective Cognitive Ability in Older

2 Adults: A Two-Year Follow-up Study

3 Author names and affiliations: Shang-Ti Chen $\mathrm{PhD}^{1}$, Clare Stevinson $\mathrm{PhD}^{2}$, Tien Tien

$4 \quad \mathrm{PhD}^{3}$, Li-Jung Chen $\mathrm{PhD}^{4^{*}}$, Po-Wen $\mathrm{Ku} \mathrm{PhD}{ }^{1,5,6^{*}}$

$5{ }^{1}$ Graduate Institute of Sports and Health, National Changhua University of Education,

6 Changhua, Taiwan

$7 \quad{ }^{2}$ School of Sport, Exercise, and Health Sciences, Loughborough University,

8 Leicestershire, United Kingdom

$9{ }^{3}$ Postgraduate Department, Guangdong Polytechnic Normal University, Guangzhou,

10 China

$11{ }^{4}$ Department of Exercise Health Science, National Taiwan University of Sport,

12 Taichung, Taiwan

$13{ }^{5}$ Department of Kinesiology, National Tsing Hua University, Hsinchu, Taiwan

$14{ }^{6}$ Institute of Public Health and Community Medicine Research Center, National

15 Yang-Ming University, Taipei, Taiwan

16 Corresponding authors:

17 Po-Wen $\mathrm{Ku}, \mathrm{PhD}^{*}$

18 Affiliation: Graduate Institute of Sports and Health, National Changhua University of

19 Education, Taiwan

20 Address: No.1, Jin-De Rd., Changhua City, 500 Taiwan 50007

21 Email: powen@cc.ncue.edu.tw

22 Li-Jung Chen, $\mathrm{PhD}^{*}$

23 Affiliation: Department of Exercise Health Science, National Taiwan University of

24 Sport, Taiwan

25 Address: No. 16, Section 1, Shuangshi Road, Taichung City, Taiwan 404 
1 Email: ljchen@gm.ntupes.edu.tw

2

\section{Highlights:}

4 - More daily steps were linearly related to a reduced rate of subjective cognitive 5 decline

6 - 3,500-6,999 steps/day was related to an approximate reduction of $40 \%$ in the rate $7 \quad$ of subjective cognitive decline.

8 - 7000 steps/day was related to an approximate reduction of $60 \%$ in the rate of 9 subjective cognitive decline. 


\section{Abstract}

2 There is still a paucity of longitudinal studies examining the relationships between

3 objectively-assessed daily steps and cognitive performance in older adults. The current study

4 aimed to explore whether there is a dose-response relationship between accelerometer-

5 measured daily steps and subjective cognitive decline rate after 2 years in older adults. A

6 total of 285 community-dwelling older adults (age $=74.52 \pm 6.12$ years, female $=55.4 \%$ )

7 wore accelerometers for 7 consecutive days measuring daily steps in 2012. Subjective

8 cognitive ability was measured using a Chinese version of the Ascertain Dementia 8-item

9 Questionnaire (AD8). In total 274 (96.1\%) participants completed the follow-up study in

10 2014. Multivariable negative binomial regression adjusted for confounders was undertaken.

11 Daily steps were linearly related to a reduced decline rate in subjective cognitive ability after

122 years. When daily steps were categorized into groups $(<3,500,3,500-6,999$, and $\geq 7,000$

13 steps/day), taking approximately 3,500-6,999 steps/day was associated with a reduced

14 subjective cognitive decline rate $(\mathrm{RR}=0.57,95 \% \mathrm{CI}=0.37-0.89)$ after 2 years compared

15 with $<3,500$ steps/day. When accruing $\geq 7,000$ steps/day, the decline rate progressively

16 decreased further $(R R=0.43,95 \% \mathrm{CI}=0.23-0.82)$. Sensitivity analyses supported the

17 stability of these findings. These results suggest that there is an inverse dose-response association of daily steps with subjective cognitive decline rate. Even as few as 3,500-6,999 steps/day was associated with a lower subjective cognitive decline rate after 2 years.

20 Accumulating $\geq 7,000$ steps/day could provide greater protection for subjective cognitive 21 ability. 


\section{Introduction}

Subjective cognitive decline in later life is a serious public health concern. Subjective cognitive decline refers to the self-reported perception of worsening cognitive abilities (e.g., memory loss) (Centers for Disease Control and Prevention, 2020) and has been recognized as a marker future cognitive impairment (Jessen et al., 2014). According to the Alzheimer's Association, the annual costs of cognitive decline for Americans aged over 65 years in 2019 is $\$ 290$ billion; these costs are projected to increase by more than $\$ 1.1$ trillion in 2050 (Alzheimer's Association, 2019). Older adults with cognitive decline including Alzheimer's disease are at greater risk of independence loss, low quality of life, and premature mortality compared with those who are cognitively intact (Fiest et al., 2016). Given the potential impact of deleterious health consequences and healthcare expenditure caused by cognitive decline, it is vital to identify the underlying factors for protecting older adults' cognitive health.

A physically active lifestyle has demonstrable benefits on older adults' health outcomes, including cognitive functioning (Ku, Chen, \& Xu, 2016; Murphy et al., 2018; UK Chief Medical Officers, 2019; U.S. Department of Health and Human Services, 2018). In particular, walking is the most common daily activity contributing to a physically active lifestyle among older adults (Rafferty et al., 2002). In general, older adults take between 2,000 and 9,000 steps a day; the broad range reflecting the natural diversity of physical function (Tudor-Locke et al., 2011) as well as various motivational factors (e.g., personality traits) (Sutin et al., 2016) in older adults.

A physical activity goal of 10,000 steps per day is often encouraged (Torjesen, 2011). However, there has been little evidence to support the goal of 10,000 steps per day for improving older adults' health outcomes. For example, one review demonstrated that direct estimates of minimal amounts of free-living moderate-to-vigorous physical activity are 
1 approximately 7,000-8,000 steps a day, suggesting that this may be a reasonable threshold of

2 free-living physical activity that is also associated with current public health guidelines (i.e.

3150 minutes of MVPA a week) (Tudor-Locke et al., 2011). More recently, findings from a 3-

4 year follow-up study of 16741 women with a mean age of 72 years revealed a reduction in

5 mortality rates in association with a higher number of steps accrued up to approximately

67,500 steps per day, above which the rates plateaued (Lee et al., 2019). These findings

7 suggest that 7,000-8,000 steps a day could yield health benefits.

To date, there is only one study showing that pedometer-assessed daily steps are

9 positively associated with aspects of cognitive ability (e.g., executive functioning and

10 attention) in older age (Calamia et al., 2018). Although the study found positive associations

11 between accumulated steps and older adults' cognitive abilities, there are gaps in the

12 literature that calls for more research. First, it is a cross-sectional study, which cannot

13 establish causal temporality between daily steps and cognitive ability in older adults. Second,

14 although pedometers are often utilized in daily steps measurement, uni-axial pedometers are

15 less sensitive to detect movements than tri-axial accelerometry, and are especially prone to

16 underestimating step counts (Dijkstra et al., 2008). Third, it is important to consider the

17 device wear time as a covariate for examining the relations between objectively-assessed

18 physical activity parameters and health outcomes (Ku et al., 2019) because failure to take

19 absolute wear time into account means that estimates of daily steps may vary according to the

20 duration of objective recordings. Finally, the dose-response association of daily steps and

21 cognitive ability in older adults remains unclear; there is limited evidence on the number of

22 daily steps needed to have a protective influence on future cognitive decline. Understanding

23 if there is a dose-response relationship based on daily steps is crucial for clinical practitioners

24 and public health guideline. 
To fill these gaps in the literature, the current study aimed to examine the doseresponse relationships between accelerometer-measured daily steps and subjective cognitive ability among community-dwelling older adults over a 2-year follow-up period. We also included underlying factors in multivariable analyses for adjustment and performed sensitivity analyses to test for confounding and reverse causation.

\section{Materials and Methods}

\subsection{Sample and Study Design}

The is a two-year longitudinal study of a community-based project in Hunei District, Kaohsiung, the second largest city in Taiwan. The first wave of measures (baseline) was conducted between August and October 2012. Participants in the current study were recruited via quota sampling (national distribution of population according to gender and age in 2011) (Taiwan Ministry of Interior, 2017). Of the 14 villages in the district, approximately 20 participants were recruited from each community center at each village. Participants in the current study were recruited via quota sampling (national distribution of population according to gender and age in 2011) (Taiwan Ministry of Interior, 2017). Recruitment posters were posted on community center bulletin boards 2 weeks before interviewing. Inclusion criteria in this study were community-dwelling older adults aged 65 years or above. In contrast, exclusion criteria in this study were defined as individuals who cannot walk by themselves due to physical limitations or severe chronic diseases. At baseline, 285 community-dwelling older adults (age $=74.52$ years, age range $=65.0-97.0$ years, female $=55.4 \%$ ) were recruited through standardized face-to-face interviews. Demographic, health status, cognitive ability data were collected during interviews and they were asked to wear an accelerometer for 7 consecutive days. The second wave of measures (follow-up) was conducted between May to July 2014 (after an average of $22.12 \pm 1.46$ months) during which 274 participants $(96.1 \%$ ) completed interviews on cognitive ability. 
A post-hoc power analysis for negative binomial regression models was conducted using version 15.0 of Power Analysis and Sample Size Software (NCSS 2017, Kaysville, UT) for the prospective analysis. Using maximum likelihood estimation, the total sample of 274 participants achieved $91.73 \%$ power at a 0.05 significance level to detect an incidence rate ratio of 0.50 (i.e. the average rate ratio based on the adjusted models in the section of Results).

The study protocol was reviewed and approved by National Taiwan University of Sport Institutional Review Board ethical committee (reference number: NTUPES-HSC-10009). All participants provided written informed consent before commencing the study.

\subsection{Measures}

2.2.1. Subjective Cognitive ability. A Chinese version of the Ascertain Dementia 8item Questionnaire (AD8) contains 8 items measuring subjective cognitive decline (Galvin et al., 2005; Galvin et al., 2007). The 8 items assessed a change in the last several years caused by cognitive problems (i.e., problems with judgment, less interest in hobbies/activities, repeating the same things over and over, difficulty learning how to use a tool or appliance, forgetting correct month or year, difficulty handling complicated financial affairs, difficulty remembering appointments, and daily problems with thinking and/or memory). Participants rated changes in their cognitive decline across four domains (memory, orientation, problemsolving abilities, and daily activities) on a scale from 0 to 8 , with higher scores indicating worse subjective cognitive ability. The AD8 has been shown to have acceptable reliability and validity among community-dwelling Taiwanese older adults (Yang et al., 2011). Our results indicated that the Cronbach's alpha of AD8 in the current sample ranged from 0.79 (baseline) to 0.81 (follow-up).

2.2.2. Daily steps. Daily steps were assessed using a triaxial accelerometry (GT3X, ActiGraph, Pensacola, FL, USA). Participants were instructed to wear the accelerometer on 
1 their waist attached by an elastic belt on 5 weekdays and 2 weekend days for 7 consecutive

2 days. The average accelerometer's wear time was $14.95(S D=2.98)$ hours per day. To

3 include the accelerometer data in the analysis 5 days of recordings with a minimum of 10

4 hours of monitoring time each day were required (Koolhaas et al., 2017). If the requirements

5 were not fulfilled, participants were requested to re-wear the accelerometer (Ku et al., 2017).

6 Five participants encountered a problem in the use of accelerometer due to running out of

7 battery and were requested to re-wear the accelerometer. To explore the dose-response

8 relationship between daily steps and cognitive ability, continuous steps per day and

9 categories of steps per day $(<3,500,3,500-6,999, \geq 7,000$ steps/day) were both analyzed. The

10 cut-off-points for the categories were based on the recommendation by Tudor-Locke et al.

11 (2011).

2.2.3. Covariates. The following factors at baseline were selected as covariates based on previous studies (Abbott et al., 2004; Calamia et al., 2018): (1) socio-demographic factors: gender, age, education attainment, and marital status; (2) lifestyle behavior: accelerometermeasured moderate-to-vigorous physical activity (MVPA) (<150 vs. $\geq 150 \mathrm{~min}$. per week) (U.S. Department of Health and Human Services, 2018), accelerometer-measured sedentary time (ST) $(<9,9-10.99,11+$ hours a day) (Ku et al., 2019), smoking, and drinking; (3) health status: body mass index (BMI) $(<18.50,18.50-23.99,24-26.99,27+)$ (Taiwan Department of Health, 2003), number of chronic diseases (none, 1, 2+), including hypertension, stroke,

20 diabetes, heart disease, cancer, chronic obstructive pulmonary disease, liver disease, renal

21 disease, and arthritis, depressive symptoms (yes/no), Activities of daily living (ADL; no

22 difficulty at all versus some or great difficulties), and baseline AD8 scores; (4) mean daily 23 accelerometer wear time.

\section{2.3. Data Analysis}


2.3.1. Descriptive statistics. Descriptive statistics were conducted to describe the characteristics of study sample. One-way ANOVA tests were used to test group differences in subjective cognitive ability at follow-up across step categories and covariates at baseline. We included the variables that showed significant group differences $(p<0.05)$ in subjective cognitive ability at follow-up in the subsequent multivariable regression models for adjustment.

2.3.2. Partial correlations. To reduce the potential confounding effect of accelerometer wear time, partial correlations were carried out to explore the associations between daily steps (continuous and categorical) and cognitive ability in 2012 (first-wave) and 2014 (second-wave) after adjusting for mean daily accelerometer wear time.

2.3.3. Negative binomial regression. Negative binomial regression was used because the outcome variable in the study was highly skewed and over-dispersed based on the Lagrange multiplier test (Norušis, 2011). Two separate multivariable regression models were conducted to assess the prospective association between daily steps (continuous and categorical) and cognitive ability after adjusting for socio-demographic, health status, baseline cognitive ability, and mean daily accelerometer wear time. To assess the possibility of multi-collinearity, the associations between daily steps and covariates were assessed using Spearman's rank correlations and partial correlations adjusted for mean daily accelerometer wear time. The correlation coefficients ranged between 0.14 and 0.51 (all less than 0.80), suggesting that multi-collinearity was not a problem (Field, 2017).

2.3.4. Sensitivity analyses. Sensitivity analyses were performed to evaluate confounding and to test the robustness of the relations between categorical steps and cognitive ability. In Model 1 and 2, we included daily steps (categorical) and daily steps (continuous) at baseline in the multivariable models respectively to assess the association of baseline daily steps with subjective cognitive ability at follow-up. We have further performed 
1 a regression model which included the quadratic term of daily steps into the fully adjusted

2 model (i.e. Model 2). However, only the linear term $(p=0.03)$ instead of the quadratic term

$3(p=0.29)$ was significant. Therefore, this indicates that there is a linear relationship between

4 daily steps and subjective cognitive ability at follow-up measures. Then, we mitigated the

5 potential influences on the relations by excluding the participants with ADL difficulty $(n=$

6 13), depressive symptoms (i.e. GDS scores equal or greater than 5) $(n=49)$, and subjective

7 cognitive decline (i.e. AD8 scores equal or greater than 2) respectively (Guo et al., 1988).

Data analyses were all conducted using IBM SPSS 22.0 (Chicago, IL). Statistical

9 significance was evaluated at $\alpha=0.05$.

3. Results

\section{3.1. Characteristics}

Group differences in subjective cognitive ability at follow-up categorized by baseline descriptive statistics of participants are presented in Table 1. Participants were more likely to

14 have lower subjective cognitive ability at follow-up if they were female, older, unmarried,

15 had lower educational levels, lower daily steps, greater ST, engaged in MVPA less than 150

16 minutes a week, had more chronic diseases, had depressive symptoms, and experienced difficulty in activities at baseline (all $p$-values $<0.05$ ). On average, daily steps were $4,733.91$ $(S D=3,073.56)$ in community-dwelling older adults. Only $17.5 \%$ of participants walked over 7,000 steps in daily life and $41.2 \%$ of participants walked less than 3,500 steps a day. 
1 Table 1

2 Subjective Cognitive Ability (AD8) at Follow-Up according to Socio-Demographic and Other Factors

$3 \quad(n=274)$

\begin{tabular}{|c|c|c|c|c|}
\hline Baseline factors & $\mathrm{n}$ & $\begin{array}{l}\text { AD8 scores } \\
\text { Mean }(S D)\end{array}$ & $p$-value ${ }^{\mathrm{a}}$ & $\begin{array}{c}\text { Effect size } \\
\text { (Eta-squared) }\end{array}$ \\
\hline \multicolumn{5}{|l|}{ Socio-demographic } \\
\hline Sex & & & 0.015 & 0.02 \\
\hline Male & 125 & $0.85(1.31)$ & & \\
\hline Female & 149 & $1.29(1.61)$ & & \\
\hline Age & & & $<0.001$ & 0.04 \\
\hline $65-69$ & 70 & $0.64(0.87)$ & & \\
\hline $70-79$ & 146 & $1.12(1.55)$ & & \\
\hline $80+$ & 58 & $1.55(1.81)$ & & \\
\hline Educational attainment & & & $<0.001$ & 0.04 \\
\hline Junior high school or above & 48 & $0.46(0.82)$ & & \\
\hline Elementary school & 113 & $1.17(1.49)$ & & \\
\hline No formal schooling & 113 & $1.27(1.66)$ & & \\
\hline Marital status & & & 0.010 & 0.03 \\
\hline Married or cohabitating & 192 & $0.92(1.37)$ & & \\
\hline Others & 82 & $1.48(1.71)$ & & \\
\hline \multicolumn{5}{|l|}{ Lifestyle behaviors } \\
\hline Steps per day & & & $<0.001^{\mathrm{b}}$ & 0.09 \\
\hline $7,000+$ & 49 & $0.49(1.43)$ & & \\
\hline $3,500-6,999$ & 112 & $0.82(1.43)$ & & \\
\hline$<3,500$ & 113 & $1.61(1.45)$ & & \\
\hline MVPA ( $\geq 150 \mathrm{~min} /$ week) & & & $0.006^{\mathrm{b}}$ & 0.03 \\
\hline Yes & 35 & $0.45(1.47)$ & & \\
\hline No & 239 & $1.18(1.47)$ & & \\
\hline ST (hours/day) & & & $0.004^{\mathrm{b}}$ & 0.04 \\
\hline$<9$ & 151 & $0.80(1.72)$ & & \\
\hline 9-10.99 & 68 & $1.08(1.47)$ & & \\
\hline $11+$ & 55 & $1.92(1.93)$ & & \\
\hline Smoking & & & 0.088 & 0.02 \\
\hline No & 261 & $1.03(1.47)$ & & \\
\hline Yes & 13 & $1.59(1.74)$ & & \\
\hline Drinking & & & 0.588 & 0.001 \\
\hline No & 261 & $1.08(1.49)$ & & \\
\hline Yes & 13 & $1.31(1.75)$ & & \\
\hline \multicolumn{5}{|l|}{ Health Status } \\
\hline Weight status (body mass index, BMI) & & & 0.966 & $<0.001$ \\
\hline Normal and underweight $(<23.99)$ & 112 & $1.18(1.31)$ & & \\
\hline Overweight (24-26.99) & 101 & $1.12(1.70)$ & & \\
\hline Obese $(27+)$ & 61 & $1.07(1.47)$ & & \\
\hline Number of chronic diseases & & & 0.026 & 0.03 \\
\hline None & 118 & $0.86(1.30)$ & & \\
\hline 1 & 104 & $1.13(1.37)$ & & \\
\hline $2+$ & 52 & $1.52(2.00)$ & & \\
\hline Depressive symptoms & & & 0.022 & 0.02 \\
\hline No & 225 & $0.99(1.41)$ & & \\
\hline Yes & 49 & $1.53(1.78)$ & & \\
\hline Activities of daily living (ADL) & & & 0.005 & 0.03 \\
\hline
\end{tabular}


No difficulty at all 261

Some or great difficulties

$13 \quad 2.23(2.13)$

1 Notes. MVPA = Moderate-to-vigorous physical activity. ST $=$ Sedentary time. ${ }^{a}$ One-way analysis of variance. ${ }^{\mathrm{b}}$ One-way analysis of covariance (adjusting for mean daily accelerometer wear time).

\subsection{Correlations between Daily Steps and Subjective Cognitive Ability at Follow-up}

Table 2 displays partial correlations between baseline daily steps (including both continuous and categorical variables) and subjective cognitive ability at baseline and followup. Briefly, participants with more daily steps at baseline were cross-sectionally and

9 longitudinally inversely related to cognitive decline after adjusting for mean daily

10 accelerometer wear time $(p<0.05)$. Additionally, Figure 1 displays associations of

11 categorical daily steps $(<3,500,3,500-6,999, \geq 7,000$ steps/day) with subjective cognitive

12 ability at baseline and follow-up.

13 Table 2

14 Associations of Baseline Daily Steps with Subjective Cognitive Ability at Follow-Up Adjusting for

Mean Daily Accelerometer Wear Time Using Partial Correlation Analyses $(n=274)$

\begin{tabular}{|c|c|c|c|c|}
\hline \multirow{2}{*}{ Variables } & \multicolumn{3}{|c|}{ Baseline } & \multirow{2}{*}{$\frac{\text { Follow-up }}{4}$} \\
\hline & 1 & 2 & 3 & \\
\hline 1. Daily steps (categorical) ${ }^{\mathrm{a}}$ & 1.00 & & & \\
\hline 2. Daily steps (continuous) ${ }^{\mathrm{b}}$ & $0.86^{* * *}$ & 1.00 & & \\
\hline 3. Cognitive ability (baseline) & $-0.17 * *$ & $-0.17 * *$ & 1.00 & \\
\hline 4. Cognitive ability (follow-up) & $-0.29 * * *$ & $-0.27 * * *$ & $0.46^{* * * *}$ & 1.00 \\
\hline
\end{tabular}
Notes. ${ }^{\text {a }}$ Low $(<3,500$ steps $)=1$, medium $(3,500-6,999$ steps $)=2$, high $(7,000+$ steps $)=3 .{ }^{b}$

17 Every increment of 1000 steps. A higher score of AD8 indicated worse subjective cognitive

18 ability. ${ }^{*} p<0.05, * * p<0.01$, *** $p<0.001$ 


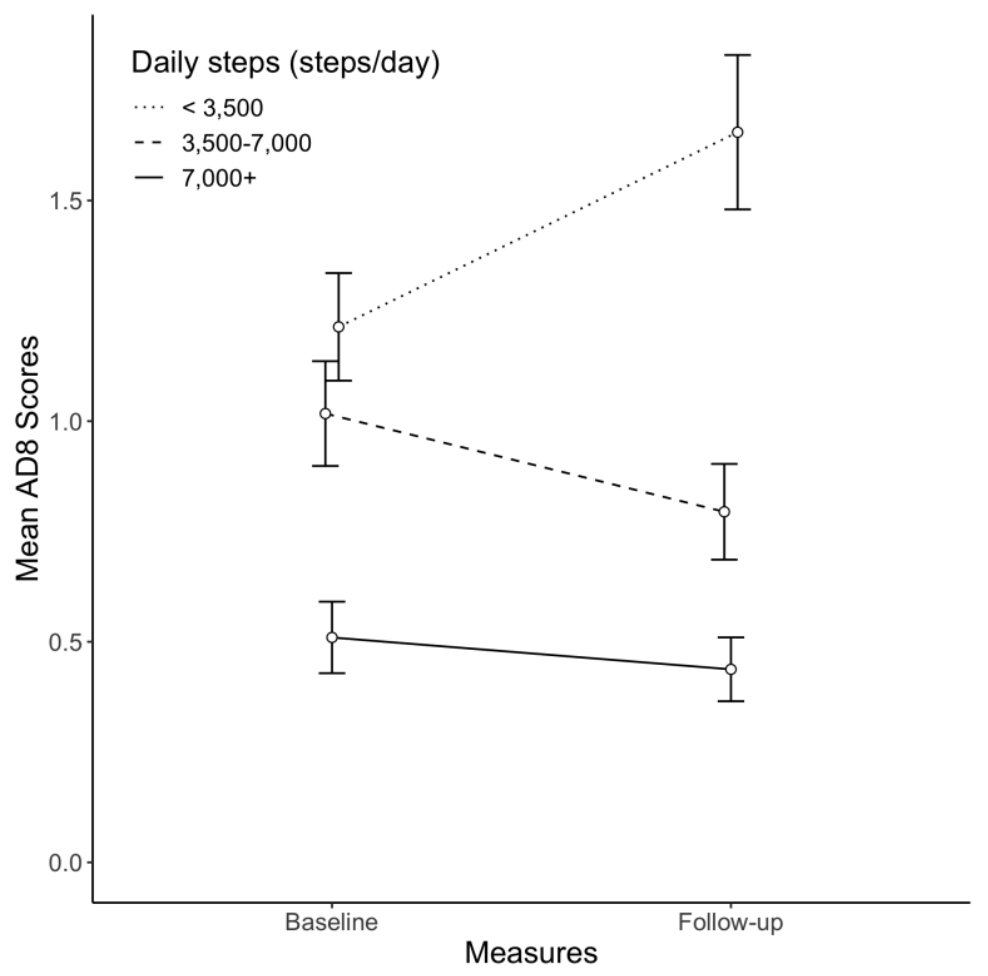

2 Figure 1

3 Associations of Categorical Daily Steps with Subjective Cognitive Ability at Baseline and Follow-up $4 \quad(n=274)$

$5 \quad$ Notes. A higher score of AD8 indicated worse subjective cognitive ability.

7 3.3. Multivariate Relations between Daily Steps and Subjective Cognitive Ability at

8 Follow-up

In multivariable-adjusted regression models testing step categories in everyday life as

10 predictors, participants with 3,500-6,999 steps/day (rate ratio $[\mathrm{RR}]=0.57,95 \% \mathrm{CI}=0.37$ -

$110.89)$ and $\geq 7,000$ daily steps $(\mathrm{RR}=0.43,95 \% \mathrm{CI}=0.23-0.82)$ were significantly associated

12 with reduced subjective cognitive rate decline at follow-up compared with $<3,500$ steps/day

13 after adjusting for all covariates (Table 3, Model 1). The subjective cognitive decline rate was

14 observed to decline progressively with those having 7000 or more steps a day. Model 2

15 included daily steps as a continuous variable and suggested that daily steps were linearly

16 associated with subjective cognitive decline in older adults $(\mathrm{RR}=0.89,95 \% \mathrm{CI}=0.83 .-0.97)$. 
$1 \quad$ Table 3

2 Negative Binomial Regression Models for Rate Ratios of Levels of Daily Steps for Predicting

3 Subjective Cognitive Ability at Follow-up $(n=273)$

\begin{tabular}{|c|c|c|}
\hline & Model 1 & Model 2 \\
\hline Variables at baseline & Rate Ratio (95\% CI) & Rate Ratio (95\% CI) \\
\hline \multicolumn{3}{|l|}{ Socio-demographic } \\
\hline \multicolumn{3}{|l|}{ Sex } \\
\hline Male & $0.78(0.49-1.23)$ & $0.78(0.49-1.22)$ \\
\hline Female & 1.00 & 1.00 \\
\hline \multicolumn{3}{|l|}{ Age } \\
\hline $65-69$ & $0.66(0.36-1.20)$ & $0.67(0.37-1.23)$ \\
\hline $70-79$ & $0.86(0.54-1.37)$ & $0.85(0.54-1.36)$ \\
\hline $80+$ & 1.00 & 1.00 \\
\hline \multicolumn{3}{|l|}{ Education level } \\
\hline Junior high school or above & $0.56(0.29-1.10)$ & $0.60(0.31-1.17)$ \\
\hline Elementary school & $1.28(0.80-2.03)$ & $1.26(0.79-2.00)$ \\
\hline No formal schooling & 1.00 & 1.00 \\
\hline \multicolumn{3}{|l|}{ Marital status } \\
\hline Married or cohabitating & $0.71(0.48-1.06)$ & $0.74(0.50-1.10)$ \\
\hline Others & 1.00 & 1.00 \\
\hline \multicolumn{3}{|l|}{ Lifestyle behaviors } \\
\hline \multicolumn{3}{|l|}{ Steps per day (categorical) } \\
\hline $7,000+$ & $0.43(0.23-0.82)^{* *}$ & \\
\hline $3,500-6999$ & $0.57(0.37-0.89)^{* *}$ & \\
\hline$<3,500$ & 1.00 & \\
\hline Steps per day (continuous) ${ }^{\mathrm{a}}$ & & $0.89(0.83-0.97)^{* *}$ \\
\hline \multicolumn{3}{|l|}{ Health Status } \\
\hline \multicolumn{3}{|l|}{ Number of chronic diseases } \\
\hline None & $0.77(0.46-1.29)$ & $0.73(0.44-1.20)$ \\
\hline 1 & $0.74(0.45-1.23)$ & $0.70(0.43-1.15)$ \\
\hline $2+$ & 1.00 & 1.00 \\
\hline \multicolumn{3}{|l|}{ Depressive symptoms } \\
\hline No & $0.76(0.48-1.18)$ & $0.75(0.48-1.17)$ \\
\hline Yes & 1.00 & 1.00 \\
\hline \multicolumn{3}{|l|}{ Activities of daily living } \\
\hline No difficulty at all & $0.54(0.24-1.18)$ & $0.47(0.21-1.04)$ \\
\hline Some or great difficulties & 1.00 & 1.00 \\
\hline Baseline AD8 scores & $1.34(1.17-1.54)^{* *}$ & $1.32(1.16-1.52)^{* *}$ \\
\hline
\end{tabular}

4 Notes. A higher score of AD8 indicated worse subjective cognitive ability. Mean daily

5 accelerometer wear time was included into each model. ${ }^{a}$ Every increment of 1000 steps. ** $p$

$6<0.01$

7

8 3.4. Sensitivity Analyses

9

The sensitivity analyses examining the confounding and reverse causation bias

10 support the relationships between more daily steps and reduced rate of subjective cognitive 
1 decline at two-year follow-up. As shown in Table 4, the patterns of results from Model 3 to

2 Model 9 remained similar when adjusting for ST or MVPA as well as excluding participants

3 with ADL difficulty or participants with depressive symptoms or subjective cognitive decline

4 at baseline. Notably, there was a borderline significant trend $(p=0.086)$ for daily steps after

5 ST and MVPA were further included in the same model.

6 Table 4

7 Sensitivity Analyses for Examining the Effect of Baseline Daily Steps on Subjective Cognitive Ability

8 at Follow-up

\begin{tabular}{|c|c|c|}
\hline Models & Rate Ratio $(95 \% \mathrm{CI})$ & $p$-value \\
\hline \multirow{2}{*}{\multicolumn{2}{|c|}{$\begin{array}{l}\text { Model } 3(\mathrm{n}=274) \\
\text { Steps per day }\end{array}$}} & \multirow{5}{*}{0.040} \\
\hline & & \\
\hline $7,000+$ & $0.46(0.23-0.93)$ & \\
\hline $3,500-6,999$ & $0.62(0.39-0.98)$ & \\
\hline$<3,500$ & 1.00 & \\
\hline \multicolumn{3}{|l|}{ Model $4(n=274)$} \\
\hline Steps per day & & 0.029 \\
\hline $7,000+$ & $0.50(0.22-1.10)$ & \\
\hline $3,500-6,999$ & $0.58(0.37-0.90)$ & \\
\hline$<3,500$ & 1.00 & \\
\hline \multirow{2}{*}{\multicolumn{2}{|c|}{$\begin{array}{c}\text { Model } 5(\mathrm{n}=274) \\
\text { Steps per day }\end{array}$}} & \multirow{5}{*}{0.086} \\
\hline & & \\
\hline $7,000+$ & $0.52(0.22-1.22)$ & \\
\hline $3,500-6,999$ & $0.62(0.39-0.98)$ & \\
\hline$<3,500$ & 1.00 & \\
\hline \multicolumn{3}{|l|}{ Model $6(n=260)$} \\
\hline Steps per day & & \multirow[t]{4}{*}{0.013} \\
\hline $7,000+$ & $0.43(0.21-0.85)$ & \\
\hline $3,500-6,999$ & $0.58(0.37-0.91)$ & \\
\hline$<3,500$ & 1.00 & \\
\hline \multicolumn{3}{|l|}{ Model $7(n=224)$} \\
\hline Steps per day & & \multirow[t]{4}{*}{0.007} \\
\hline $7,000+$ & $0.35(0.16-0.76)$ & \\
\hline $3,500-6,999$ & $0.55(0.34-0.89)$ & \\
\hline$<3,500$ & 1.00 & \\
\hline \multicolumn{3}{|l|}{ Model $8(n=208)$} \\
\hline Steps per day & & \multirow[t]{4}{*}{0.030} \\
\hline $7,000+$ & $0.43(0.21-0.89)$ & \\
\hline $3,500-6999$ & $0.52(0.30-0.93)$ & \\
\hline$<3,500$ & 1.00 & \\
\hline \multicolumn{3}{|l|}{ Model $9(n=171)$} \\
\hline Steps per day & & 0.025 \\
\hline $7,000+$ & $0.37(0.15-0.93)$ & \\
\hline $3,500-6999$ & $0.45(0.23-0.85)$ & \\
\hline
\end{tabular}


Notes. A higher score of AD8 indicated a worse subjective cognitive ability. Baseline covariates in all models: sex, age, education, marital status, number of chronic diseases, activities of daily living, depressive symptoms, AD8 scores, and mean daily accelerometer wear time.

Model 3 = Based on the model 1 in table 3, further including sedentary time

Model $4=$ Based on the model 1 in table 3, further including moderate-to-vigorous physical activity

Model 5 = Based on the model 4, further including sedentary time

Model $6=$ Based on the model 1 in table 3, excluding participants with ADL difficulty at baseline

Model $7=$ Based on the model 1 in table 3, excluding participants with depressive symptoms at baseline (GDS scores equal or greater than 5)

Model $8=$ Based on the model 1 in table 3, excluding participants with cognitive impairment at baseline (AD8 scores equal or greater than 2)

Model $9=$ Based on the model 6, further excluding participants with depressive symptoms, and cognitive impairment at baseline

\section{Discussion}

This prospective cohort study investigated the dose-response associations between accelerometer-assessed daily steps and subjective cognitive ability in community-dwelling older adults over a two-year follow-up. Our findings suggested that more daily steps taken in free-living conditions were linearly associated with a reduced subjective cognitive decline rate. When daily steps were further categorized into three levels $(<3,500,3,500-6,999$, and $\geq$ 7,000 steps per day), older adults who averaged approximately 3,500-6,999 steps/day had a significantly lower subjective cognitive decline rate compared with those who took less than 3,500 steps a day. With at least 7,000 steps a day, the rate of decline progressively decreased further. These prospective associations were robust even when adjusting for underlying covariates including socio-demographic, health status, baseline subjective cognitive ability, 
1 and mean daily accelerometer wear time. Sensitivity analyses for testing confounding effects and reverse causation further supported the findings.

The mean daily step count of participants was 4,733 in this study, which was slightly higher than those aged over 60 years (approximately 4,000 steps/day) in 111 countries across the globe as collected by smartphone accelerometers (Althoff et al., 2018). The difference may be due to daily steps being underestimated by a smartphone because people may not carry a smartphone in all domains of their everyday routine. Additionally, the range of daily steps in this study (1,660-7,807 steps/day) is similar to Tudor-Locke et al.'s (2011) results showing that mean daily steps in U.S. older adults are 2,000-9,000. The broad range of daily steps reflects the diversity of a physically active lifestyle among older adults. Consistent with Calamia et al.'s (2018) study using pedometers, our findings suggested that daily steps were linearly associated with cognitive decline rate among older adults. Our finding is in similar with Spartano et al.'s (2019) findings that middle-aged adults (aged 53 years) who took more than 7,500 steps or more per day were related to higher brain volume, compared to those without meeting PA guidelines (150 minutes of MVPA a week). Additionally, our findings expand on the conclusions of a recent systematic review (Keily et al., 2018) to suggest that the cognitive decline rate was reduced by approximately $40 \%$ for older adults who took more than 3,500 steps a day and approximately $60 \%$ for those reaching at least 7,000 steps a day. The suggestion that reaching approximately 7,000 steps/day prevented the decline in older adults' cognitive health seems to be consistent with prior studies (Lee et al., 2019; Tudor-Locke et al., 2011), showing that older adults with approximately 7,000 steps/day at baseline had decreased rates of cardiovascular mortality and all-cause mortality. Our findings demonstrate that older adults could target 3,500 steps/day as a preliminary goal to protect cognitive health; they could further target more than 7,000 steps/day as an advanced goal to receive greater protection from future cognitive impairment. 
Walking is a common daily activity in older adults. There is no established mechanism to explain the associations between daily steps monitored in free-living conditions and older adults' cognitive health. It may be related to the effects of aerobic exercises on brain function through a neuropsychological mechanism. Previous studies have demonstrated that older adults showed a significant increase in brain structure and gray matter volume after participating in aerobic exercises training protocol (Colcombe et al., 2006; Erickson, Leckie, \& Weinstein, 2014). In addition, a meta-analysis has demonstrated that engaging in aerobic exercises may elicit up-regulation of brain-derived neurotrophic factor (BDNF), neocortical modifications, and increases in hippocampal volume (Firth et al., 2018). Given that taking daily steps is one of the activities that contributes to aerobic energy expenditure (Tudor-Locke et al., 2011), older adults who engage in more daily steps tend to gain more cardiovascular and neural benefits from physical activity., This can help prevent age-related hippocampal deterioration and maintain cognitive health compared with those who engage in a lower volume of daily steps.

In addition, it is possible that older adults engaging in more MVPA are more likely to accumulate steps taken in free-living conditions. In contrast, those who live in a sedentary lifestyle tend to spend more time in prolonged sitting and therefore have fewer daily steps. However, we have further included potential confounding variables (i.e. the status of MVPA and sedentary time) for adjustment in sensitivity analyses, demonstrating that the association patterns did not alter. These results indicated that daily steps are prospectively associated with cognitive ability, which is independent of the effects of MVPA and sedentary time. A major strength of our study is its prospective design and use of tri-axial accelerometry. Pedometer-assessed steps may significantly decrease accuracy for detecting short trajectories (lower than 5 meters) and slow walking pace (Dijkstra et al., 2008). This study could detect daily steps with higher accuracy than those based on pedometers. 
1 Furthermore, device wear time can confound analyses on objective physical activity and

2 health outcome (Ku et al., 2019), and the present research is able to avoid this by taking mean

3 daily accelerometer wear time into account. Additionally, we also utilized multiple regression

4 models with a range of covariates to confirm the effects of daily steps on subsequent

5 cognitive ability when adjusting for MVPA and ST, which have been identified as potential

6 confounders in the relationship between daily steps and cognitive ability (Barkrania et al.,

7 2018). Several sensitivity analyses were also performed to reduce the possibility of reverse

8 causality.

Despite these strengths, there are some limitations that should be considered. First, the current study was based on community-dwelling older adults using a sex-age quota sampling with a satisfactory response rate on follow-up, but there may still be problems of sample representativeness. Compared with a previous research based on the Taiwan National Health Interview Survey, it revealed that there were no significant differences in educational attainment, but that participants in the current study had fewer chronic conditions, and less difficulty in ADL (data not shown) (Chen, Stevinson, Ku, Chang, \& Chu, 2012). Because physical inactivity and cognitive decline may be less prevalent in this healthier subgroup of the population, potential selection bias may have led to underestimation of the relationships of daily steps with rate of cognitive decline. Second, the AD8 is a briefly self-rating tool that may not accurately represent concurrent levels of comprehensive cognitive abilities as measured by objective cognitive tests (e.g., Repeatable Battery for the Assessment of

21 Neuropsychological Status). Information on the AD8 may only suggest individuals' subjective cognitive abilities. Thus, future longitudinal research should for instance to explore the relationship between accelerometer-measured daily steps and cognitive abilities with objective measures in older age. Third, causality cannot be determined due to the observational nature of the data. To strengthen causal inferences and better inform 
1 interventions regarding the effects of daily steps on cognitive ability in older adults,

2 experimental designs are required for future studies. Finally, the scope of the step categories

3 examined in this study may not represent the entire spectrum of walking patterns to fully

4 understand the dose-response patterns. Research shows that various dimensions of walking

5 patterns such as step speed, step length, and step width may have similar but distinctive

6 influences on cognitive decline rate (Kikkert et al., 2016; Taiguchi et al., 2019).

7

8

9

\section{Conclusions}

In sum, this study expands our understanding of the dose-response associations between accelerometer-assessed daily steps taken in free-living condition and subjective cognitive ability in community-dwelling older adults. It presents evidence that even as few as 3,500-6,999 steps/day at baseline was more likely to have lower subjective cognitive decline rate over a two-year follow-up. When at least 7000 daily steps were accumulated, the rate progressively decreased further. This extends the existing evidence for the benefits of physical activity for preventing cognitive impairment in older adults.

\section{Funding}

This work was partly supported by the Taiwan Ministry of Science and Technology (MOST 104-2410-H-018 -028 and MOST 108-2811-H-018-500).

\section{Declaration of competing interest}

The authors declare that they have no conflict of interest. 


\section{References}

2 Abbott, R. D., White, L. R., Ross, G. W., Masaki, K. H., Curb, J. D., Petrovitch, H., 2004. Walking and dementia in physically capable elderly men. JAMA. 292(12), 14471453. doi:10.1001/jama.292.12.1447

Althoff, T., Sosič, R., Hicks, J. L., King, A. C., Delp, S. L., \& Leskovec, J., 2017. Largescale physical activity data reveal worldwide activity inequality. Nature. 547, 336339. doi:10.1038/nature23018.

Alzheimer's Association, 2019. Alzheimer's disease facts and figures. https://www.alz.org/media/Documents/alzheimers-facts-and-figures-2019-r.pdf

Barkrania, K., Edwardson, C. L., Khunti, K., Bandelow, S., Davies, M. J., Yates, T., 2018. Associations between sedentary behaviors and cognitive function: Cross-Sectional and prospective findings from the UK biobank. Am J Epidemiol. 187(3), 441-454. https://doi.org/10.1093/aje/kwx273

Calamia, M., De Vito, A., Bernstein, J. P. K., Weitzner, D. S., Carmichael, O. T., Keller, J. N., 2018. Pedometer-assessed steps per day as a predictor of cognitive performance in older adults. Neuropsychology. 32(8), 941-949. doi:10.1037/neu0000487

Centers for Disease Control and Prevention, 2020. Subjective cognitive decline-A public health issue. https://www.cdc.gov/aging/data/subjective-cognitive-decline-brief.html

Chen, L. J., Stevinson, C., Ku, P. W., Chang, Y. K., \& Chu, D. C., 2012. Relationships of leisure-time and non-leisure-time physical activity with depressive symptoms: A population-based study of Taiwanese older adults. Int J Behav Nutr Phys Act. 9(28), 1-10. doi:10.1186/1479-5868-9-28.

Colcombe, S. J., Erickson, K. I., Scalf, P. E., et al., 2006. Aerobic exercise training increases brain volume in aging humans. J. Gerontol. A Biol. Sci. Med. Sci. 61(11), 1166-1170. doi:10.1093/gerona/61.11.1166 
1 Dijkstra, B., Zijlstra, W., Scherder, E., Kamsma, Y., 2008. Detection of walking periods and number of steps in older adults and patients with Parkinson's disease: Accuracy of a pedometer and an accelerometry-based method. Age and Aging. 37(4), 436-441. doi:10.1093/ageing/afn097

Erickson, K. I., Leckie, R. L., Weinstein, A. M. Physical activity, fitness, and gray matter volume. Neurobiol. Aging. 35, S20-S28. doi:10.1016/j.neurobiolaging.2014.03.034

Field, A., 2017. Discovering statistics using IBM SPSS statistics $\left(5^{\text {th }}\right.$ ed.): North American Edition SAGE Publications Ltd, Thousand Oaks, CA.

Fiest, K. M., Jetté, N., Roberts, J. I., et al., 2016. The prevalence and incidence of dementia: a systematic review and meta-analysis. Can J Neurosci Nurs. 43(Suppl 1), 3-50. doi:10.1017/cjn.2016.18

Firth, J., Stubbs, B., Vancampfort, D., et al., 2018. Effect of aerobic exercise on hippocampal volume in humans: a systematic review and meta-analysis. Neuroimage. 166, 230238. doi:10.1016/j.neuroimage.2017.11.007

Galvin, J. E., Roe, C. M., Coats, M. A., Morris, J. C., 2007. Patient's rating of cognitive ability: using the AD8, a brief informant interview, as a self-rating tool to detect dementia. Arch Neurol. 64(5), 725-730. doi:10.1001/archneur.64.5.725

Galvin, J. E., Roe, C. M., Powlishta, K. K., et al., 2005. The AD8: a brief informant interview to detect dementia. Neurology. 65(4), 559-564. https://doi.org/10.1212/01.wnl.0000172958.95282.2a

Guo, N. W., Liu H. C., Wong, P. F., et al., 1988. Chinese version and norms of the MiniMental State Examination. J Clin Epidemiol. 41(10), 971-978. https://doi.org/10.1016/0895-4356(88)90034-0 
1 Jessen, F., Amariglio, R. E., van Boxtel, et al., 2014. A conceptual framework for research on subjective cognitive decline in preclinical Alzheimer's disease. Alzheimer's \& Dement. 10(6), 844-852. doi:10.1016/j.jalz.2014.01.001

Keily, P., Williamson, C., Hunter, R., Niven, A., Mutrie, N., Richards, J., 2018. Walking on sunshine: scoping review of the evidence for walking and mental health. Br J Sports Med. 52(12), 800-806. doi:10.1136/bjsports-2017-098827

Kikkert, L. H. J., Vuillerme, N., van Campen, J. P., Hortobágyi, T., Lamoth, C. J., 2016. Walking ability to predict future cognitive decline in old adults: a scoping review. Ageing Res Rev. 27, 1-14. doi:10.1016/j.arr.2016.02.001

Koolhaas, C. M., Dhana, K., van Rooij, F. J., et al., 2017. Sedentary time assessed by actigraphy and mortality: the Rotterdam Study. Prev Med. 95, 59-65. doi:10.1016/j.ypmed.2016.11.021

Ku, P. W., Chen, L. J., Xu, Z. H., 2016. A review of late-life sedentary behaviors and cognitive aging. Physical Education Journal. 49(S), 1-16. doi:10.3966/10247297201606490S001

Ku, P. W., Liu, Y. T., Lo, M. K., Chen, L. J., Stubbs, B., 2017. Higher levels of objectively measured sedentary behavior is associated with worse cognitive ability: two-year follow-up study in community-dwelling older adults. Exp Gerontol. 99, 110-114. doi:10.1016/j.exger.2017.09.014

Ku, P. W., Steptoe, A., Liao, Y., Hsueh, M.C., Chen, L. J., 2019. A threshold of objectivelyassessed daily sedentary time for all-cause mortality in older adults: a meta-regression of prospective cohort studies. J Clin Med. 8, 564. doi:10.3390/jcm8040564

Lee, I. M., Shiroma, E. C., Kamada, M., Bassett, D. R., Matthews, C. E., Buring, J. E., 2019. Association of step volume and intensity with all-cause mortality in older women. JAMA Intern Med. 179(8), 1105-1112. doi:10.1001/jamainternmed.2019.0899 
1 Murphy, M. H., Broom, D. R., Gill, J. M. R., et al., 2018. Expert working group working paper: older adults working group. UK physical activity guidelines: review and recommendations for older adults (aged 65+years). University of Bristol, UK.

Norušis, M., 2011. IBM SPSS statistics 19 advanced statistical procedures companion. Addison Wesley, Boston, MA.

Rafferty, A. P., Reeves, M. J., Mcgee, H. B., Pivarnik, J. M., 2002. Physical activity patterns among walkers and compliance with public health recommendations. Med Sci Sports. 34(8), 1255-1261. doi:10.1097/00005768-200208000-00005

Spartano, N. L., Davis-Plourde, K. L., Himali, J. J., et al. 2019. Association of Accelerometer-measured light-intensity physical activity with brain volume: The Framingham heart study. JAMA Netw Open. 2(4), e192745. doi:10.1001/jamanetworkopen.2019.2745.

Sutin, A. R., Stephan, Y., Luchetti, M., Artese, A., Oshio, A., Terracciano, A., 2016. The five-factor model of personality and physical inactivity: A meta-analysis of 16 samples. Journal of Research in Personality. 63, 22-28. doi:10.1016/j.jrp.2016.05.001

Taiguchi, Y., Watanabe, Y., Osuka, Y., et al., 2019. Characteristics for gait parameters of community-dwelling elderly Japanese with lower cognitive function. PLOS One. 14(3), e0212646. doi:10.1371/journal.pone.0212646

Torjesen, I., 2018. Sixty seconds on . . . exercise. BMJ. 362, k3006. doi:10.1136/bmj.k3006

Taiwan Department of Health, 2003. Identification, evaluation, and treatment of over-weight and obesity in adults. Taiwan Department of Health, Taipei.

Taiwan Ministry of Interior, 2017. Monthly bulletin of interior statistics: resident population by age group. Taiwan Ministry of Interior, Taipei. 
1 Tudor-Locke, C., Craig, C. L., Aoyagi, Y., et al., 2011. How many steps/day are enough? For older adults and special populations. Int J Behav Nutr Phys Act. 8, 80. doi:10.1186/1479-5868-8-80

UK Chief Medical Officers, 2019. UK Chief Medical Officers' physical activity guidelines. https://assets.publishing.service.gov.uk/government/uploads/system /uploads/attachment_data/file/829841/uk-chief-medical-officers-physical-activityguidelines.pdf

U.S. Department of Health and Human Services, 2018. Physical activity guidelines for Americans (2nd ed.). US Department of Health and Human Services, Washington, US.

Yang, Y. H., Galvin, J. E., Morris, J. C., Lai, C. L., Chou, M. C., Liu, C. K., 2011. Application of AD8 questionnaire to screen very mild dementia in Taiwanese. Am J Alzheimers Dis Other Demen. 26(2), 134-138. doi:10.1177/1533317510397330

14 Zhu, W., Howard, V. J., Wadley, V. G., et al., 2015. Association between objectively measured physical activity and cognitive function in older adults-the reasons for geographic and racial differences in stroke study. J Am Geriatr Soc. 63(12), 22472454. doi:10.1111/jgs.13829. 